\title{
Evolution of the Trigger and Data Acquisition System in the ATLAS experiment
}

\author{
Sami Kama ${ }^{1}$ \\ on behalf of the ATLAS TDAQ collaboration ${ }^{2}$ \\ ${ }^{1}$ Southern Methodist University, Texas \\ ${ }^{2}$ https://cdsweb.cern.ch/record/1386334
}

IEEE Nuclear Science Symposium and Medical Imaging Conference, 2012 


\section{Existing Trigger and DAQ system}

- Three level design

- Level 1 built with custom $\mathrm{h} / \mathrm{w}(<2.5 \mu$ s latency $)$

- Level 2 and Event Filter form High Level Trigger(HLT) and are s/w triggers running on commodity PCs

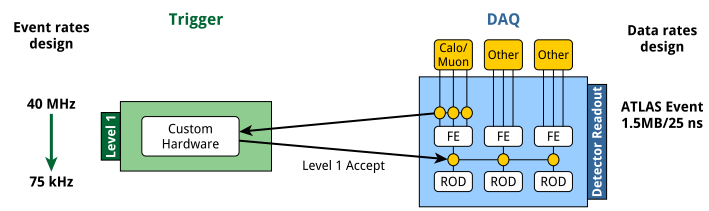




\section{Existing Trigger and DAQ system}

- On L1 accept

- Data pushed to ReadOut System(ROS) PCs

- Information on detector Regions with Interesting activity (Rol) passed to Level 2

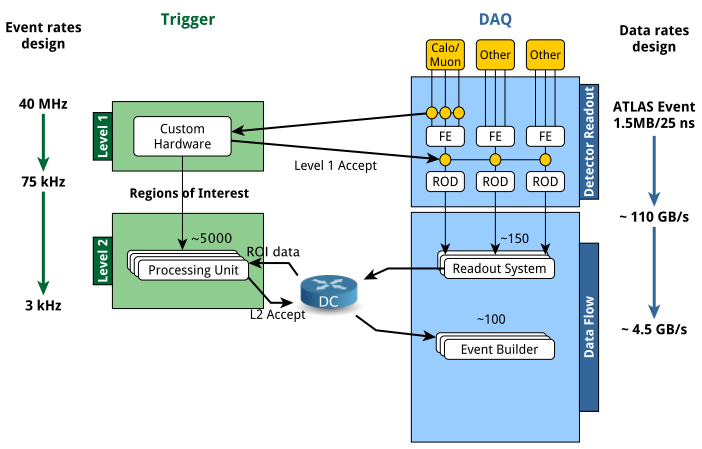




\section{Existing Trigger and DAQ system}

- Level 2 has a latency $40 \mathrm{~ms}$

- Reads only partial event data (Rol) from ROS

- L2 Accept triggers event building

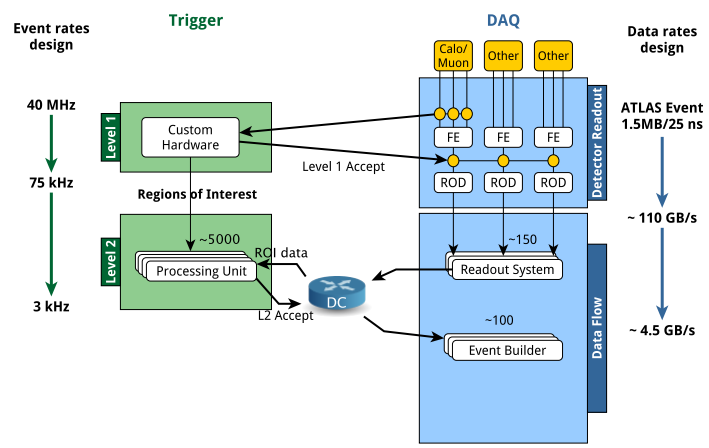




\section{Existing Trigger and DAQ system}

- Event Builder

- Event data pulled from ROS to Event Builder

- Collects full event data

- Feed assembled event data to Event Filter through BackEnd network

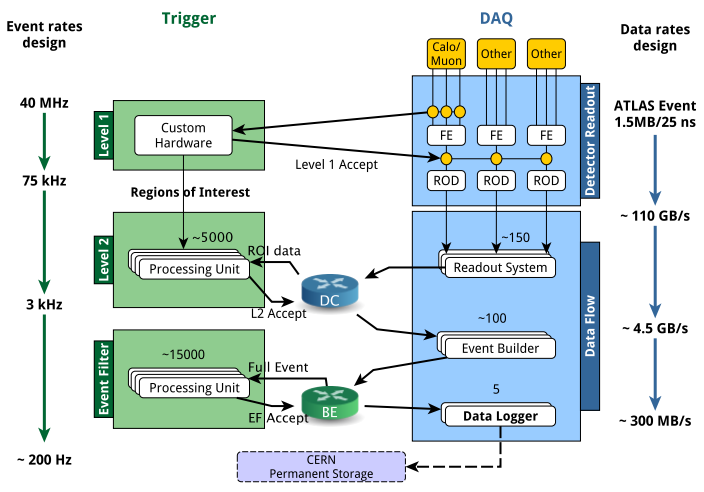




\section{Existing Trigger and DAQ system}

- Data logger

- Store events in different files (streams) depending on trigger results

- Send files to Tier 0 asynchronously

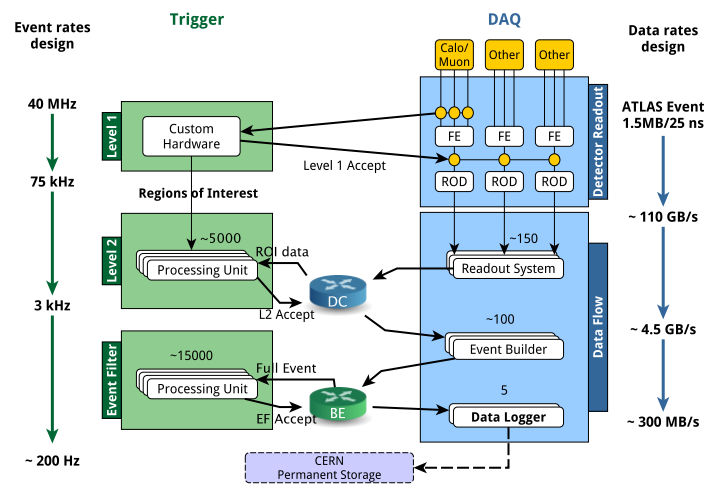




\section{Existing Trigger and DAQ system}

- Event level parallelism

- Each Processing Unit(PU) process works on single event

- Multiple processes in each node

- Operating beyond design capacity

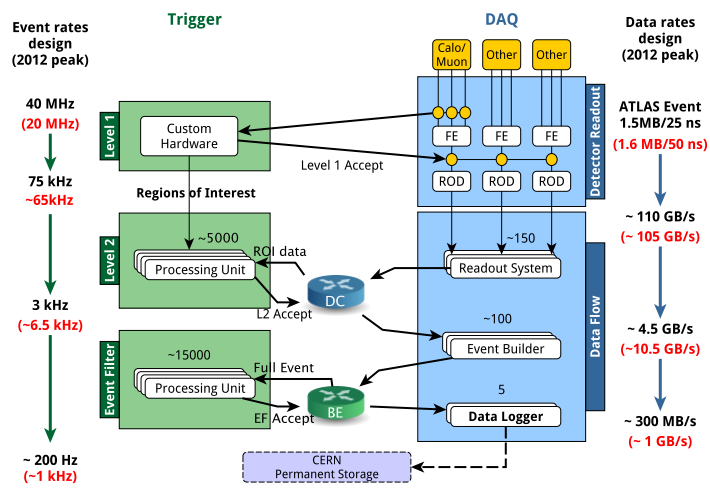




\section{Performance in 2011-2012}

- Delivered luminosity

- $5.32 \mathrm{fb}^{-1} @ 7 \mathrm{TeV}$ in 2011

- $\sim 16.4 \mathrm{fb}^{-1} @ 8$ TeV in 2012

- $93.8 \%$ efficiency in 2900

\section{hours of beam time}

- Bunch crossing is 50ns

- Average number of

interactions per bunch

crossing (pileup) in a run

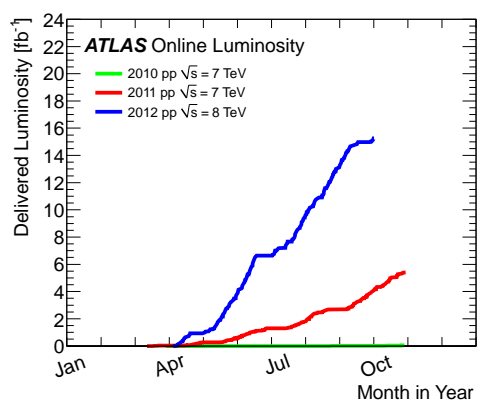

- Peak pileup already exceeded 35 interactions per bunch-crossing 


\section{Performance in 2011-2012}

- Delivered luminosity

- $5.32 \mathrm{fb}^{-1} @ 7$ TeV in 2011

- $16.4 \mathrm{fb}^{-1} @ 8 \mathrm{TeV}$ in 2012

- $93.8 \%$ efficiency in 2900 hours of beam time

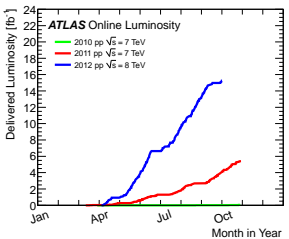

- Bunch crossing is $50 \mathrm{~ns}$

- Average number of

interactions per bunch

crossing (pileup) in a run

- 20.0 in 2012

- Peak pileup already exceeded 35 interactions per

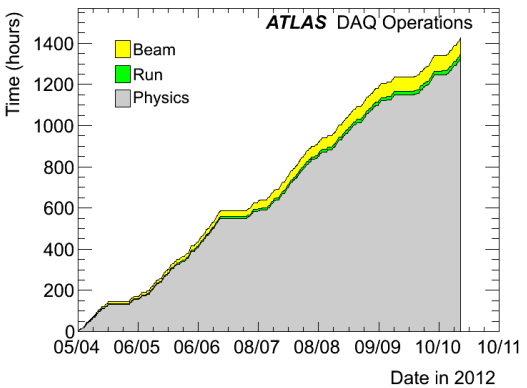

bunch-crossing

Date in 2012 


\section{Performance in 2011-2012}

- Delivered luminosity

- $5.32 \mathrm{fb}^{-1} @ 7 \mathrm{TeV}$ in 2011

- $\sim 16.4 \mathrm{fb}^{-1} @ 8 \mathrm{TeV}$ in 2012

- $93.8 \%$ efficiency in 2900 hours of beam time

- Bunch crossing is $50 \mathrm{~ns}$

- Average number of

interactions per bunch

crossing (pileup) in a run

- Peak pileup already exceeded 35 interactions per bunch-crossing
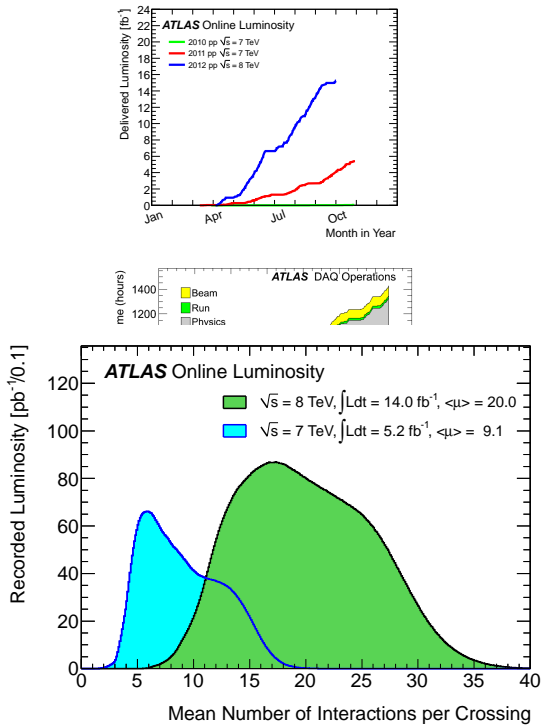


\section{Performance in 2011-2012}

- Delivered luminosity

- $5.32 \mathrm{fb}^{-1} @ 7 \mathrm{TeV}$ in 2011

- 16.4 fb $\mathrm{fb}^{-1} @ 8 \mathrm{TeV}$ in 2012

- $93.8 \%$ efficiency in 2900 hours of beam time

- Bunch crossing is $50 \mathrm{~ns}$

- Average number of interactions per bunch crossing (pileup) in a run

- 9.1 in 2011

- 20.0 in 2012

- Peak pileup already exceeded 35 interactions per
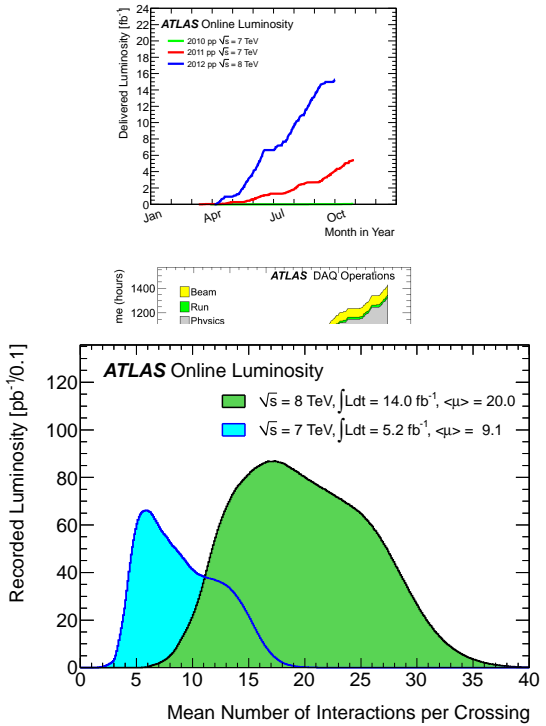


\section{Performance in 2011-2012}

- Delivered luminosity

- $5.32 \mathrm{fb}^{-1} @ 7 \mathrm{TeV}$ in 2011

- 16.4 fb $\mathrm{fb}^{-1} @ 8 \mathrm{TeV}$ in 2012

- $93.8 \%$ efficiency in 2900 hours of beam time

- Bunch crossing is $50 \mathrm{~ns}$

- Average number of interactions per bunch crossing (pileup) in a run

- 9.1 in 2011

- 20.0 in 2012

- Peak pileup already exceeded 35 interactions per bunch-crossing
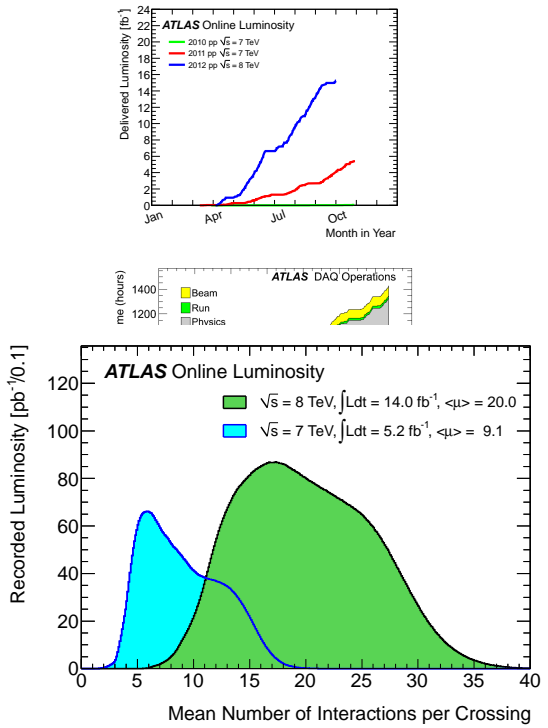


\section{Pileup Dependency}

- Event size

- Largely linear but might deviate in the future

- Recorded event size $\sim 1.6 \mathrm{MB}$

- CPU also depends on pileup

- Older nodes are approaching to their limit

- Under control until the Long

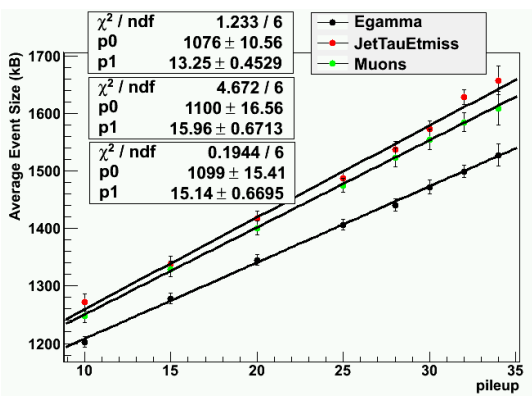
Shutdown at the beginning of next year 


\section{Pileup Dependency}

- Event size

- Largely linear but might deviate in the future

- For pileup rate of 35

- Recorded event size $\sim 1.6 \mathrm{MB}$

- Older nodes are approaching to their limit

- Under control until the Long

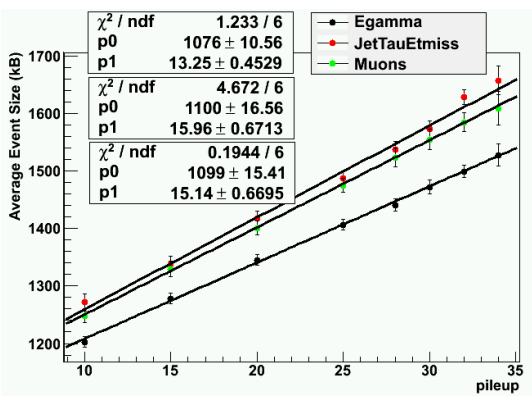
Shutdown at the beginning of next year 


\section{Pileup Dependency}

- Event size

- Largely linear but might deviate in the future

- For pileup rate of 35

- Recorded event size $\sim 1.6 \mathrm{MB}$

- CPU also depends on pileup

- Older nodes are approaching to their limit

- Under control until the Long

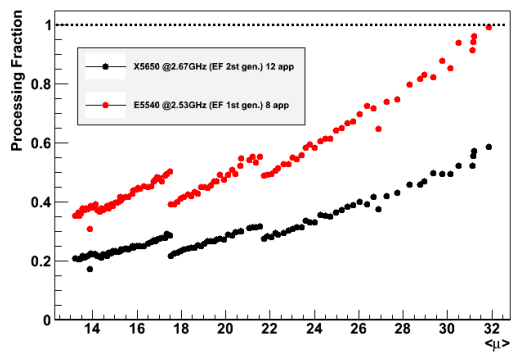
Shutdown at the beginning of next year 


\section{LHC Long Shutdown}

- Good opportunity to upgrade S/W and H/W

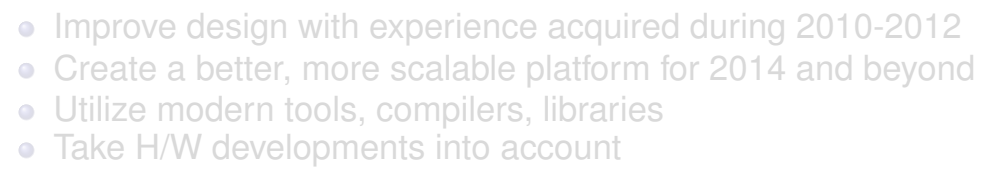




\section{LHC Long Shutdown}

- Good opportunity to upgrade S/W and H/W

- Improve design with experience acquired during 2010-2012

- Utilize modern tools, compilers, libraries

- Take H/W developments into account 


\section{LHC Long Shutdown}

- Good opportunity to upgrade S/W and H/W

- Improve design with experience acquired during 2010-2012

- Create a better, more scalable platform for 2014 and beyond 


\section{LHC Long Shutdown}

- Good opportunity to upgrade S/W and H/W

- Improve design with experience acquired during 2010-2012

- Create a better, more scalable platform for 2014 and beyond

- Utilize modern tools, compilers, libraries 


\section{LHC Long Shutdown}

- Good opportunity to upgrade S/W and H/W

- Improve design with experience acquired during 2010-2012

- Create a better, more scalable platform for 2014 and beyond

- Utilize modern tools, compilers, libraries

- Take H/W developments into account

- Many-Core systems

- GPGPUs

- Accelerator cards 


\section{LHC Long Shutdown}

- Good opportunity to upgrade S/W and H/W

- Improve design with experience acquired during 2010-2012

- Create a better, more scalable platform for 2014 and beyond

- Utilize modern tools, compilers, libraries

- Take H/W developments into account

- Many-Core systems

- GPGPUs

- Accelerator cards

- Expected parameters after shutdown

- $100 \mathrm{kHz}$ L1 Rate

- $1 \mathrm{kHz}$ EF output

- 25 ns bunch-crossing rate 


\section{Changes in Design}

- Merge L2, EB and EF into one system

- Simplify farm structure

- Simplify network topology

- All event processing in single node

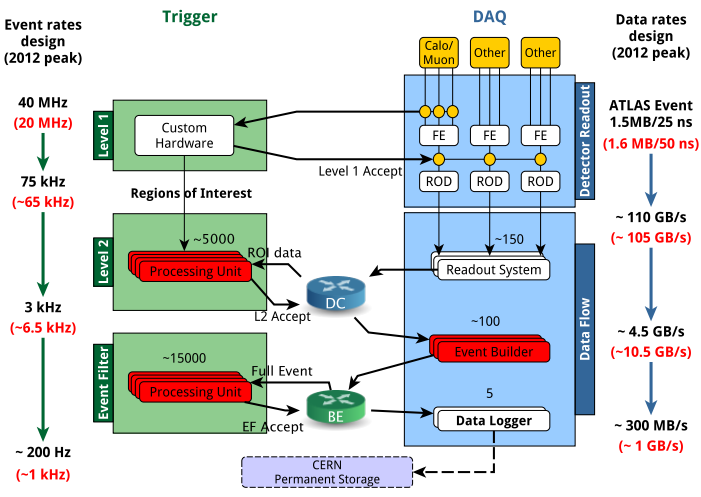




\section{Changes in Design}

- Merge L2, EB and EF into one system

- Simplify farm structure

- Simplify network topology

- All event processing in single node

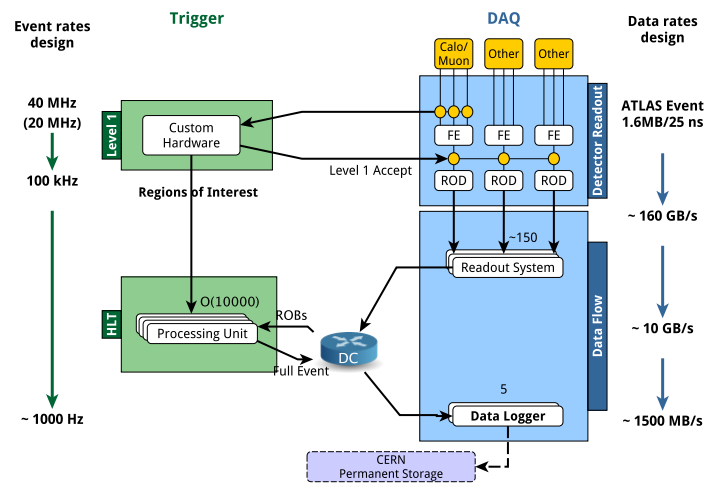




\section{Changes in Design}

- Each HLT node contains

- One Data Collection Manager (DCM)

- One or more HLT Processing Units (HLTPU)

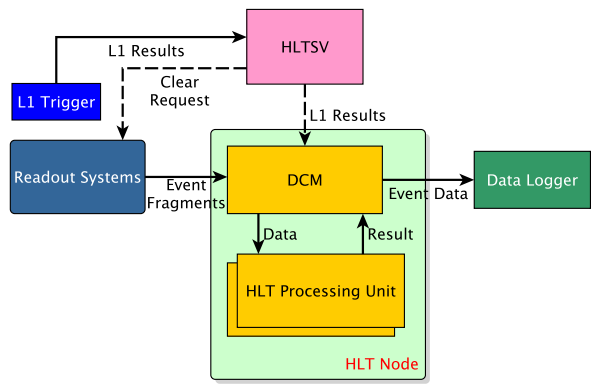




\section{Changes in Design}

- HLT Processing units

- Does event selection

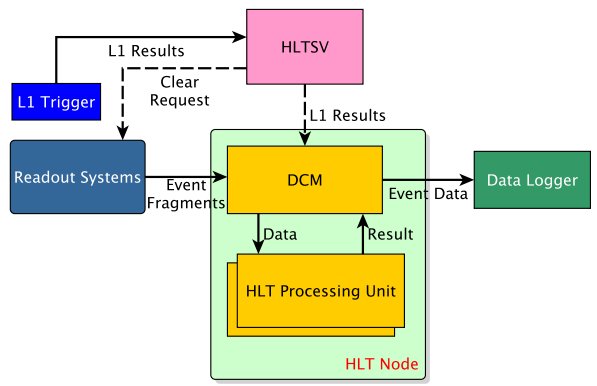




\section{Changes in Design}

- HLT Processing units

- Does event selection

- Fork multiple children

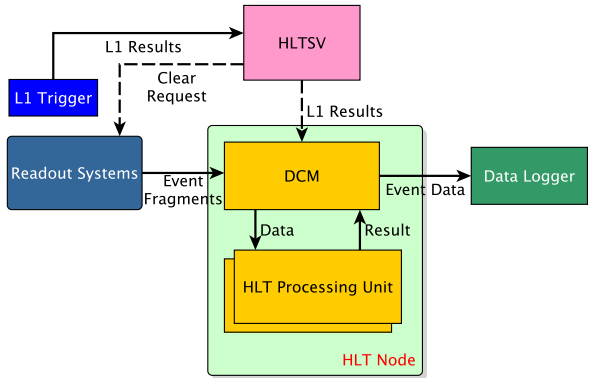




\section{Changes in Design}

- HLT Processing units

- Does event selection

- Fork multiple children

- Minimize memory utilization using Copy-on-Write feature of Linux kernel

- $\sim 300 \mathrm{MB} /$ child instead of $\sim 1.2 \mathrm{~GB} /$ process for 32bit application

Memory Utilization

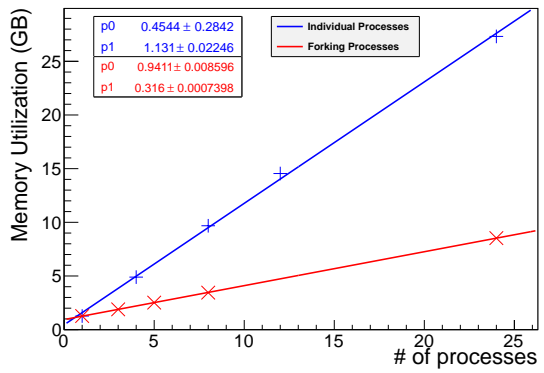




\section{Changes in Design}

- Data Collection Manager

- In charge of data collection, caching and integrity

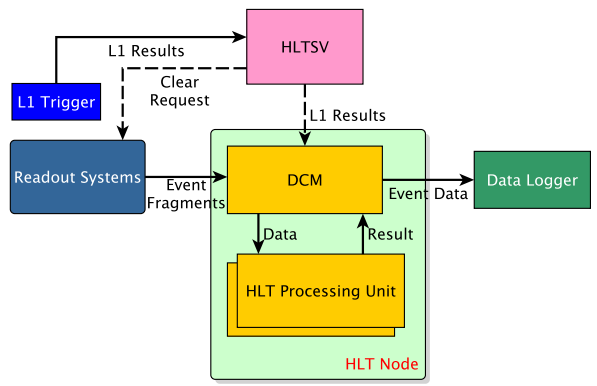




\section{Changes in Design}

- Data Collection Manager

- In charge of data collection, caching and integrity

- Assigns events to available Processing Units (HLTPU)

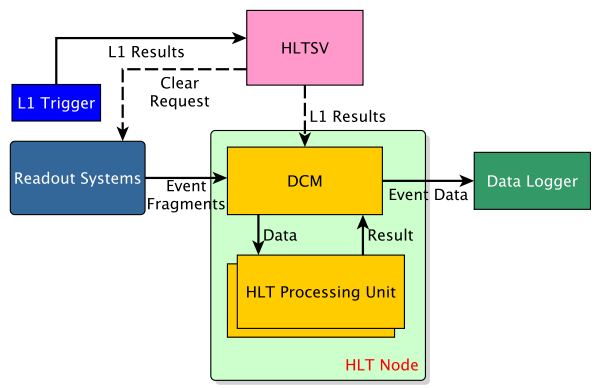




\section{Changes in Design}

- Data Collection Manager

- In charge of data collection, caching and integrity

- Assigns events to available Processing Units (HLTPU)

- Sends accepted events to Data Logger

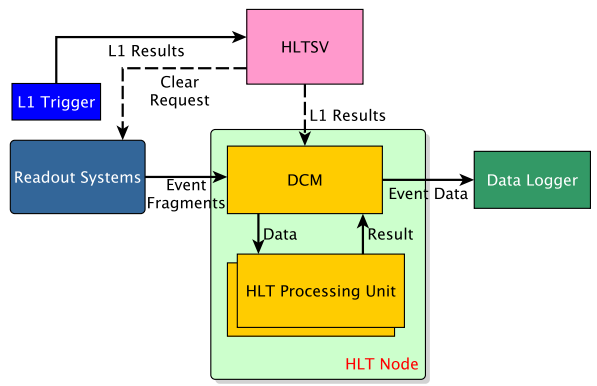




\section{Changes in Design}

- Data Collection Manager

- In charge of data collection, caching and integrity

- Assigns events to available Processing Units (HLTPU)

- Sends accepted events to Data Logger

- IPC through shared memory and unix sockets

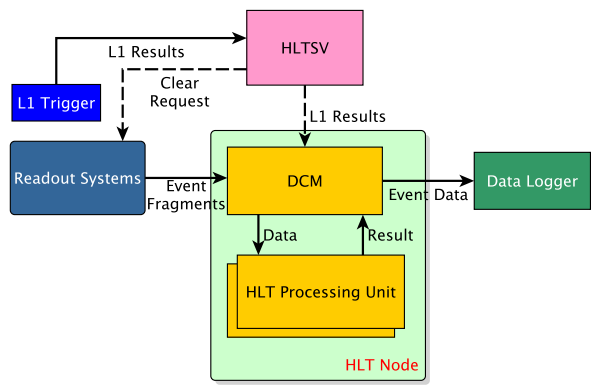




\section{Changes in Design}

- HLT SuperVisor (HLTSV) supervises HLTPU Nodes

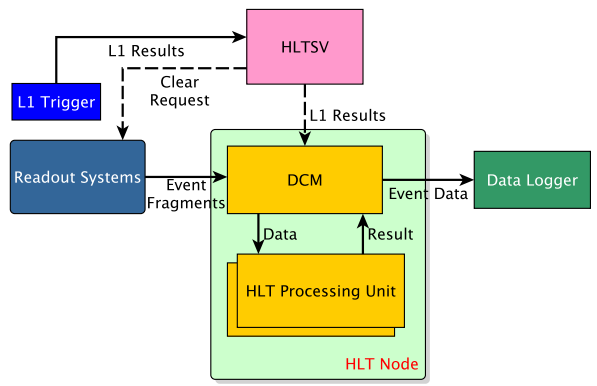




\section{Changes in Design}

- HLT SuperVisor (HLTSV) supervises HLTPU Nodes

- Gets the L1 Result from Level 1 trigger

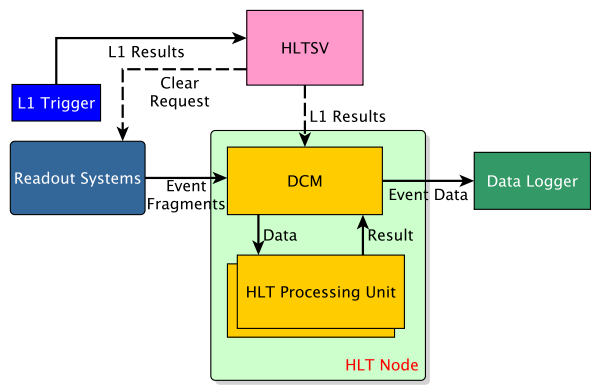




\section{Changes in Design}

- HLT SuperVisor (HLTSV) supervises HLTPU Nodes

- Gets the L1 Result from Level 1 trigger

- Assigns it to an available Data Collection Manager

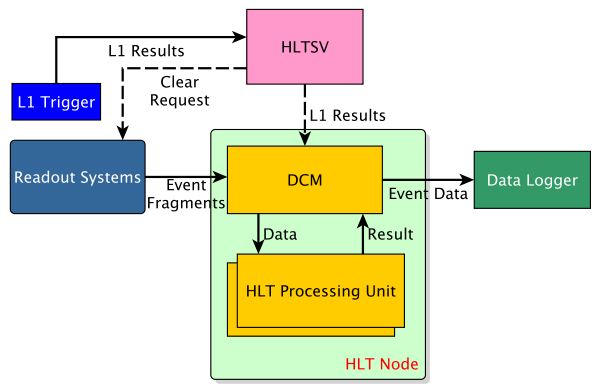




\section{Changes in Design}

- HLT SuperVisor (HLTSV) supervises HLTPU Nodes

- Gets the L1 Result from Level 1 trigger

- Assigns it to an available Data Collection Manager

- Provides load balancing of non-uniform nodes

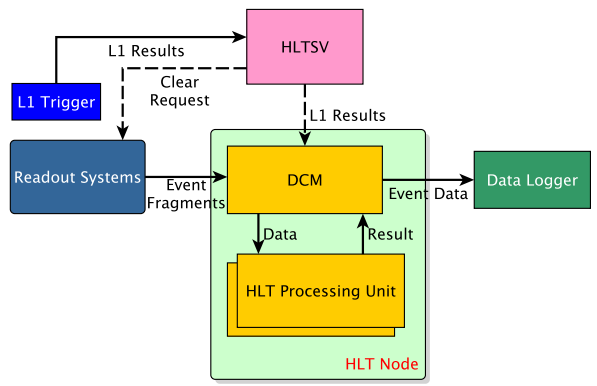




\section{Changes in Design}

- HLT SuperVisor (HLTSV) supervises HLTPU Nodes

- Gets the L1 Result from Level 1 trigger

- Assigns it to an available Data Collection Manager

- Provides load balancing of non-uniform nodes

- Single application seems enough for event rates up to $100 \mathrm{kHz}$

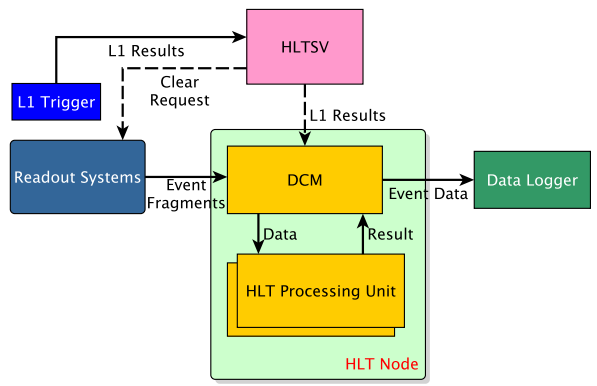




\section{Changes in Event Selection}

Current event selection

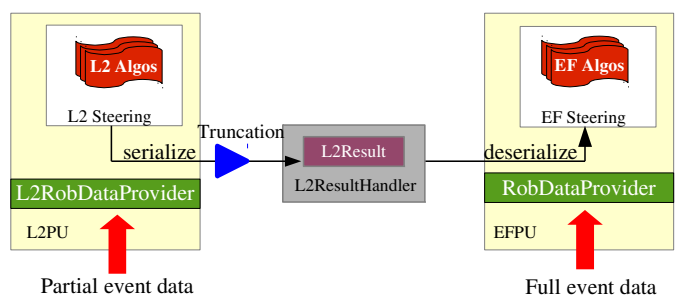

- L2PU

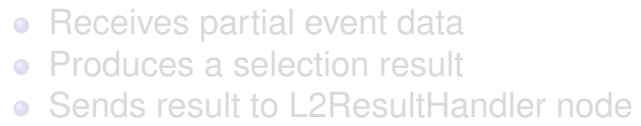

- EFPU

- Receives full event data (containing the L2Result)

- Processes the event using the L2Result as seed

- Produces final decision 


\section{Changes in Event Selection}

Current event selection

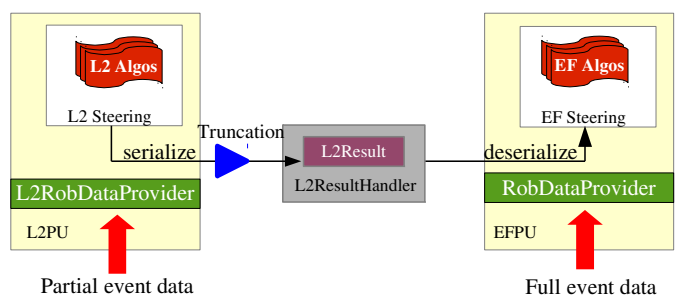

- L2PU

- Receives partial event data

- Produces a selection result

- Sends result to L2ResultHandler node

- EFPU

- Receives full event data (containing the L2Result)

- Processes the event using the L2Result as seed

- Produces final decision 


\section{Changes in Event Selection}

\section{Current event selection}

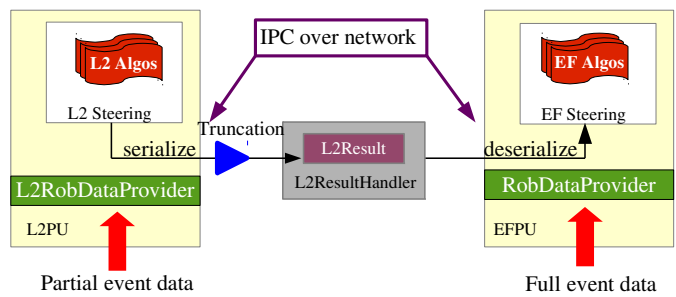

- Rol data accessed twice

- Serialization-Deserialization between processes

- Network latency

- L2 data unpacked twice 


\section{Changes in Event Selection}

Current event selection

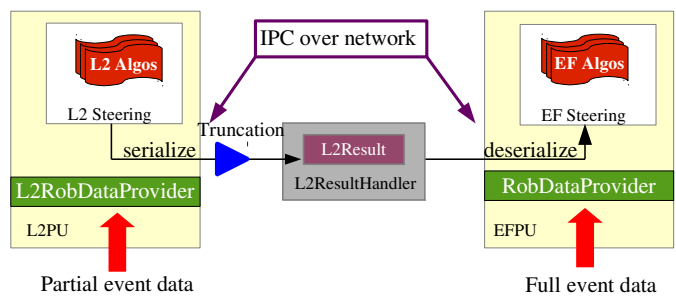

- Rol data accessed twice

- Serialization-Deserialization between processes 


\section{Changes in Event Selection}

Current event selection

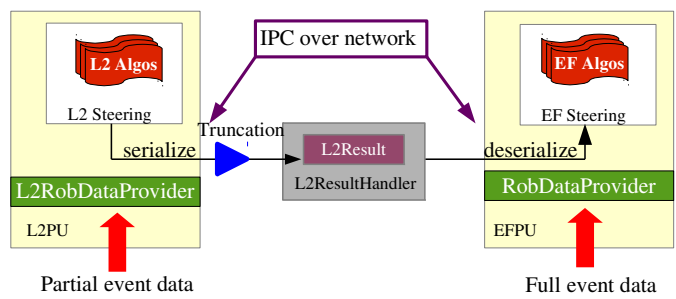

- Rol data accessed twice

- Serialization-Deserialization between processes

- Network latency 


\section{Changes in Event Selection}

Current event selection

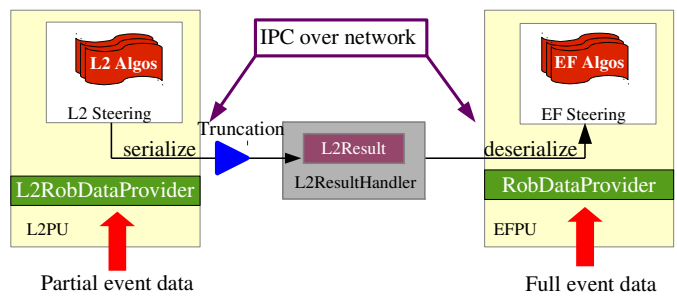

- Rol data accessed twice

- Serialization-Deserialization between processes

- Network latency

- L2 data unpacked twice 


\section{Changes in Event Selection}

Current event selection

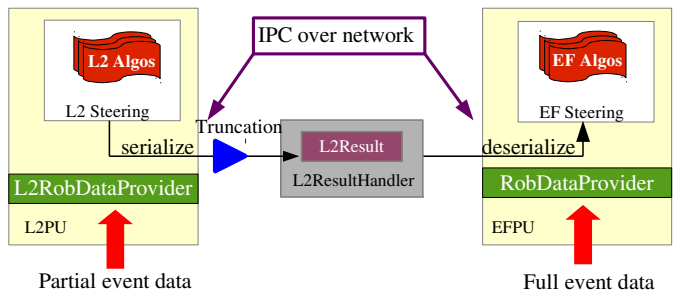

New event selection

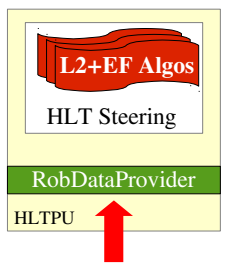

Incremental event data

- Execute L2 and EF algorithms in same process 


\section{Changes in Event Selection}

Current event selection

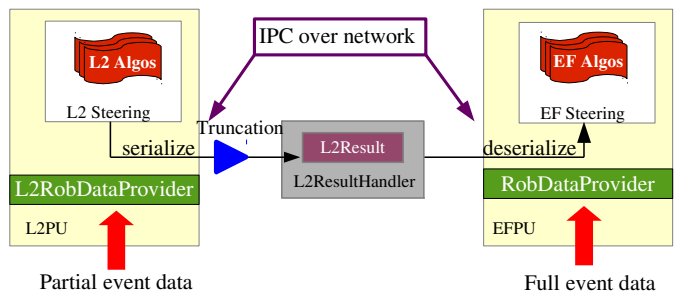

New event selection

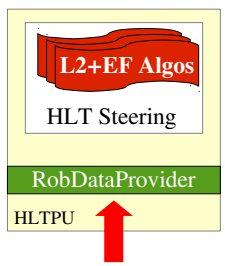

Incremental event data

- Execute L2 and EF algorithms in same process

- No latency or duplication of work

- HLT Result serialization only on accept

- Data accessed and unpacked only once 


\section{Changes in Event Selection}

Current event selection

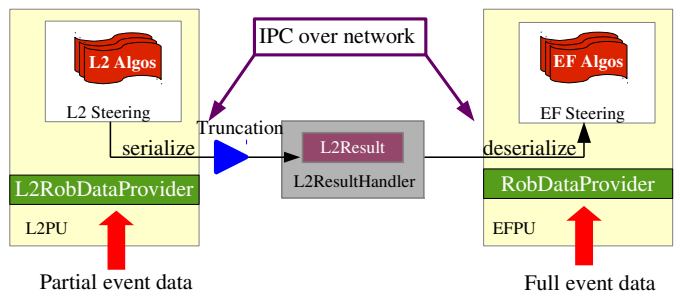

New event selection

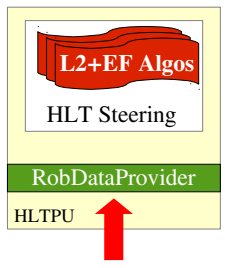

Incremental event data

- Execute L2 and EF algorithms in same process

- No latency or duplication of work

- HLT Result serialization only on accept

- Data accessed and unpacked only once

- Optimize algorithm order of execution to minimize decision time

- Execute the set of algorithms that are most likely to accept the event first

- Less load on event buffers (ROS) 


\section{Prototyping}

Prototyping efforts are ongoing

- HLTSV

- Multiple prototypes using different technologies are developed

- All exceeded $100 \mathrm{kHz}$

- DCM

- A demonstration implementation is available

- HLTPU

- Prototyping implementation is ready

- Can be used in data flow tests

- First implementations will be available at the beginning of next year

- To be tested and finalized during 2013 in test beds and at HLT farms 


\section{Summary}

- ATLAS Trigger and DAQ system has shown great performance during 2010-2012

- Current system went well beyond its design and all parameters are under control

- Evolution design will be an improvement of current system and leave the door open for future developments

- Trigger and DAQ will be ready for collecting new data after the long shutdown 


\section{Backup}

\section{HLTSV Scalability}

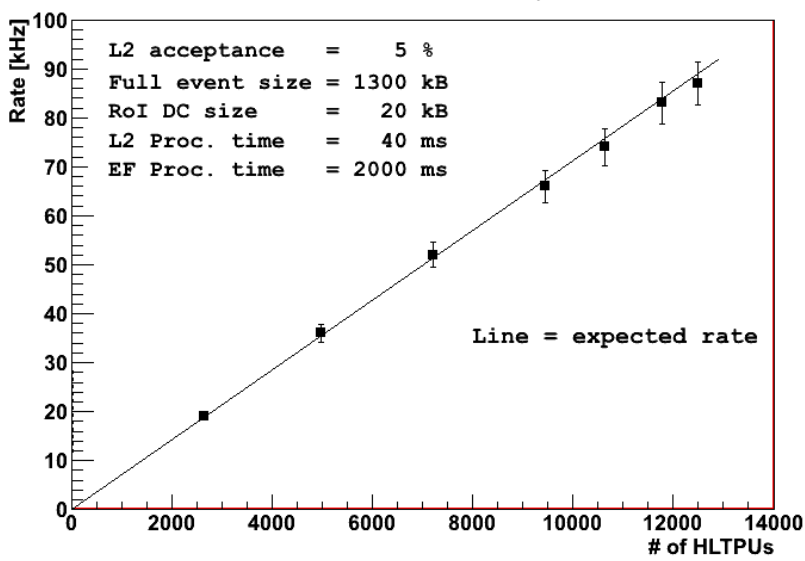

